\title{
Two-Stage Surgical Management of Multilevel Symptomatic Thoracic Haemangioma Using Ethanol and Iliac Crest Bone Graft
}

\author{
Devanand Degulmadi, Venkatramana Brahmajoshyula, Shivanand Mayi, Suman Teegala \\ Department of Orthopaedics, Kamineni Institute of Medical Sciences, Narketpally, India
}

\begin{abstract}
This article presents a 56-year-old obese female who presented with back pain and progressive weakness in her lower limbs for three months. She was bed-ridden for one week before reporting to our hospital. Plain radiographs showed vertical striations in multiple vertebrae classical of haemangioma. Magnetic resonance imaging (MRI) spine revealed multiple thoracic and lumbar vertebral haemangiomas. Extra osseous extension of haemangioma at T12 was causing spinal cord compression. Two-stage surgery was performed with absolute alcohol (ethanol) injection followed by pedicle screw fixation and decompression with tricortical iliac crest bone graft into the vertebral body. Postoperatively rapid neurological improvement was seen. After three weeks, she could walk independently. One year later, computed tomography showed complete incorporation of bone graft and maintained vertebral body height. MRI showed complete resolution of the cord edema at $\mathrm{T} 12$. These findings indicated diminished vascularity of the tumor.
\end{abstract}

Keywords: Haemangioma; Two stage surgery; Ethanol; Tricortical iliac crest bone graft

\section{Introduction}

Vertebral haemangiomas are one of the commonest benign tumours of the vertebral column, occurring mostly in the thoracic spine. The incidence has been reported as $10 \%-12 \%$ in autopsy specimens. Most haemangiomas are discovered accidentally during routine radiographic study. Only $0.9 \%-1.2 \%$ of these tumors are symptomatic. Involvement of multiple vertebrae has also been reported [1].

We discuss a unique case of multiple haemangioma of dorso-lumbar spine presenting with paraparesis and bladder involvement. Two-stage surgery with emphasis on the use of absolute alcohol (ethanol) and autogenous tricortical iliac bone strut graft to achieve structural stability are highlighted.

\section{Case Report}

A 56-year-old obese female patient presented to the outpatient department with back pain and weakness of both lower limbs for one week. She was able to walk with support, in spite of weakness for 3 months before her legs had gradually weakened to the point where she could no longer walk. The bladder was catheterized as she was unable to pass urine. Physical examination revealed severe tenderness over lower dorsal and upper lumbar spine. Motor power was $1 / 5$ in both hips and knees and 3/5 in both ankles. Reflexes were exaggerated with bilateral extensor plantar response. Sensations and distal pulses were intact.

Plain X-ray showed gross osteoporosis with vertical striations in multiple vertebral bodies characteristic of

Received Aug 24, 2013; Revised Oct 15, 2013; Accepted Nov 13, 2013

Corresponding author: Devanand Degulmadi

Department of Orthopaedics, Kamineni Institute of Medical Sciences, Narketpally, Nalgonda, 508254 Telanganaa, India

Tel: +91-9966430503, Fax: +91-8682272829, E-mail: drdanand@yahoo.co.in 
haemangioma. Magnetic resonance imaging spine revealed an expansile haemangioma of T12 vertebrae involving

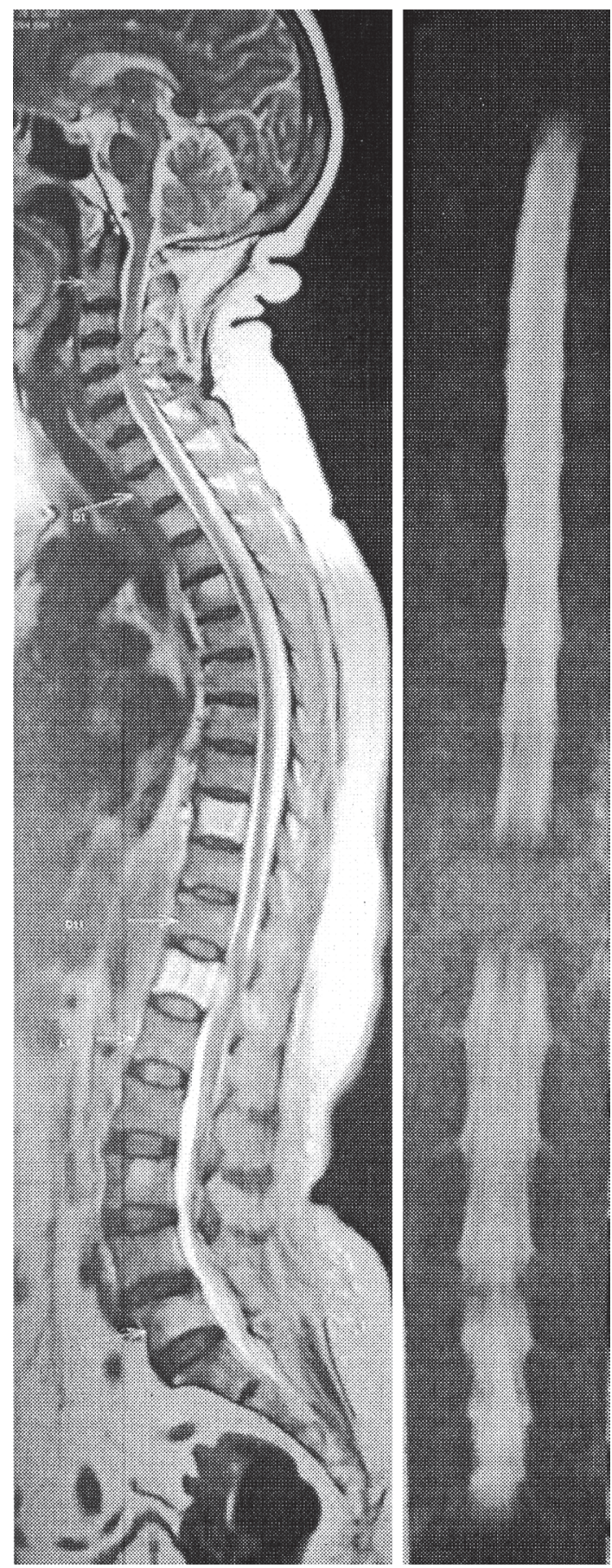

Fig. 1. Sagittal T2-weighted image of whole spine reveals increased T2 signal diffusely at the D12 vertebra with breach in posterior cortex. There is extra-osseous extension with a definite contrast-enhancing soft tissue mass extending into the spinal canal causing marked compression of the conus medullatis. Also noted a haemangioma involving D9 vertebral body with thinning of posterior cortex. There are smaller haemangiomas in D2, D4, L3, and L5 vertebral bodies. entire body with a breach in the posterior cortex causing significant cord compression. Haemangioma at the level of T9 showed a thin posterior cortex and minimal extension into pedicles (Fig. 1). Multiple thoracic (T2, T4) and lumbar (L3, L5) vertebral haemangiomas were observed.

Surgery was planned in two stages. In the first stage, absolute alcohol (ethanol) was injected into T12 vertebral body under local anaesthesia with 16 No. Jamshedi needle placed into left T12 pedicle under image guidance. Around $8 \mathrm{~mL}$ of absolute alcohol (ethanol) was injected at a very slow rate of about 1-1.5 mL every 2-3 minutes. Needle was withdrawn when blood can no longer be aspirated.

After 24 hours passed, second-stage reconstruction of the spine was performed using autogenous tricortical iliac crest bone graft combined with pedicle screws from T7 to L2 and decompression at T12 vertebral level. T9 was also included in the fixation in view of severe localized pain over the segment, gross osteoporotic quality of vertebrae, and fear of adjacent segment fracture. Thorough curettage of T12 vertebral body was performed through transpedicular route from both sides and tricortical strut bone graft placed (Fig. 2). Minimal bleeding was observed because of preoperative ethanol injection. Biopsy
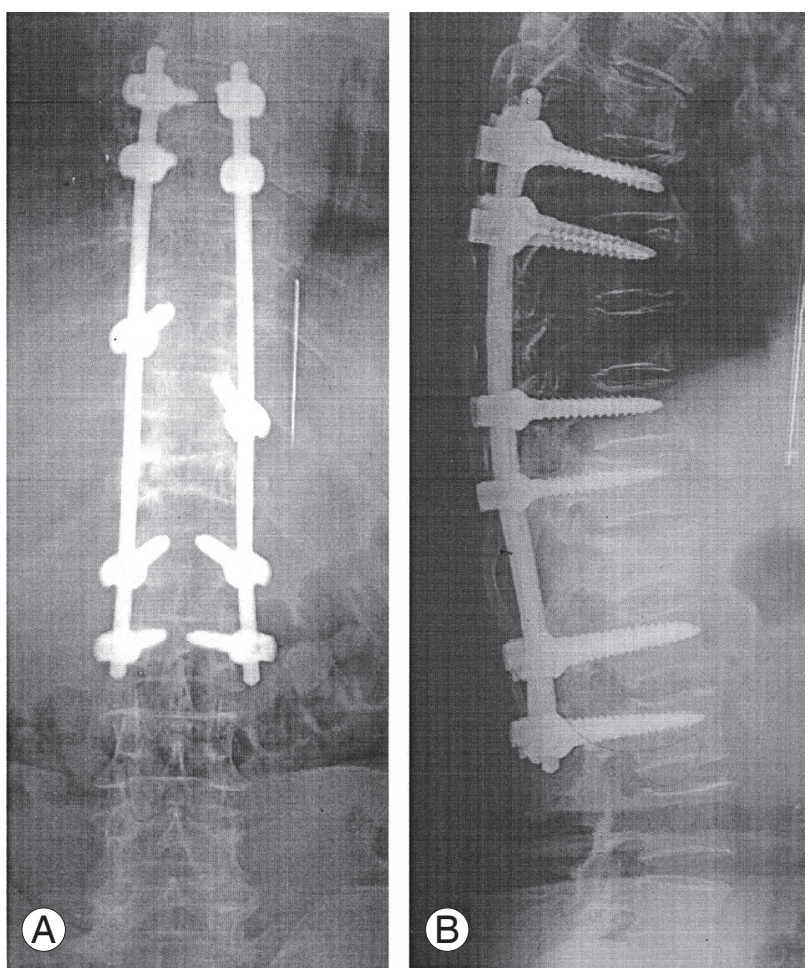

Fig. 2. Plain radiographs anteroposterior (A) and lateral (B) views taken immediately after surgery showing pedicle screw fixation from $\mathrm{D} 7$ to $\mathrm{L} 2$, bone graft placed in D12 vertebral body. 
specimens confirmed the diagnosis of haemangioma. The operative time was four hours and blood loss was 450 $\mathrm{mL}$. Postoperatively neurological improvement was noticed from fourth day onwards. Motor power improved to $4 / 5$ in three weeks and patient was mobilized with thoracolumbosacral orthosis. Bladder recovered within two weeks. Radiographs at six months postoperatively revealed no loosening of the bone graft or the pedicle screws. Computed tomography (CT) showed no absorption of iliac crest graft (Fig. 3). At one year follow-up the patient reported an almost complete absence of back pain and near-normal motor power in both lower limbs.

\section{Discussion}

Symptomatic vertebral haemangiomas are difficult to treat because of the highly vascular nature of the lesion. Four different pathophysiological mechanisms have been documented for symptomatic vertebral haemangiomas $[2,3]$; tumor extension into the epidural space was the underlying cause in our case. Distortion of the spinal canal due to tumor enlargement of the vertebral body, compression fracture, and bleeding from the mass into the epidural
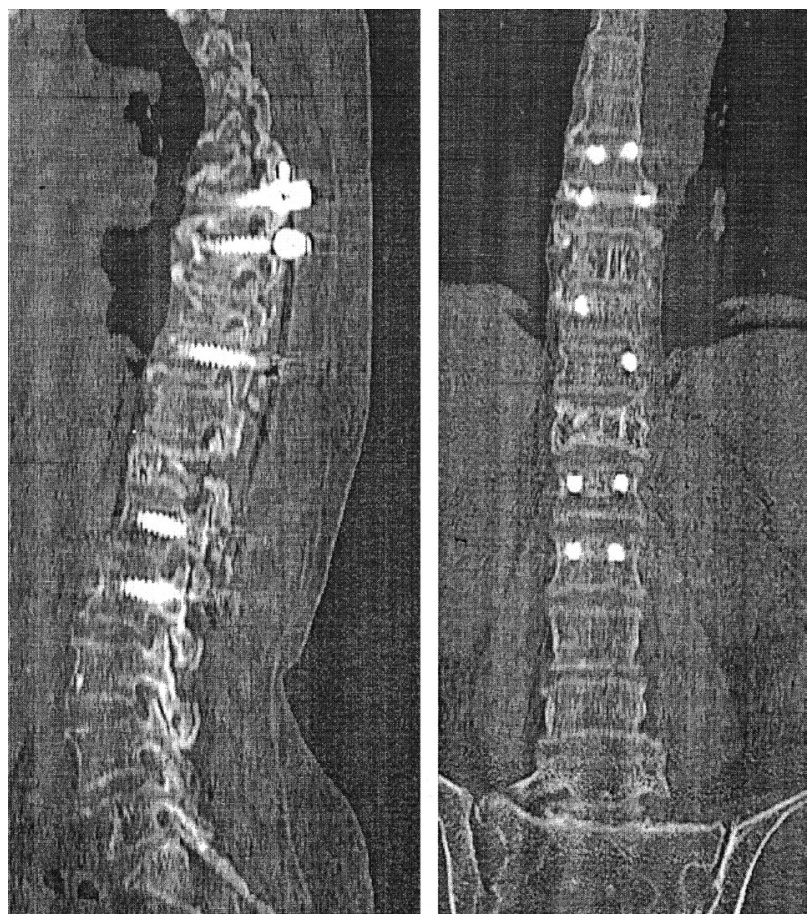

Fig. 3. At 1-year follow-up computed tomography scan images, sagittal and coronal views taken showing maintenance of vertebral body height and graft in place. space are the other three mechanisms.

Many therapeutic options, including surgery, radiotherapy, arterial embolization, and injection of methylmethacrylate into hemagiomatous vertebrae have been reported with varying degrees of success. The use of radiotherapy as a treatment modality in benign conditions like vertebral haemangioma being employed in few cases is still controversial in the fear of inherent risk of radionecrosis [4,5]. Heiss et al. [6] and Bas et al. [7] reported a successful management of spinal cord compression caused by vertebral haemangioma with intralesional injection of alcohol. Percutaneous vertebroplasty although a well documented procedure was not employed in this case as there was a breach in posterior cortex and complications in such situations are difficult to manage $[8,9]$. Though the importance of preoperative embolisation during surgery of haemangioma is well documented, as this facility was not available in our center, we choose alcohol ablation performed under Local Anesthesia preoperatively to reduce bleeding intraoperatively which is other safest method studied in the literature. This was very effective in reducing intraoperative blood loss and need for blood transfusion. Absolute ethanol induces thrombosis, edema and sclerosis; however vertebral collapse may occur leading to progressive kyphosis and mechanical insufficiency [10].

Hence, in the second stage of surgery, reconstruction of the spine was achieved by using pedicle screw fixation from T7 to L2. T9 was also included in the construct in view of severe pain localized over the segment, gross osteoporotic quality of bone and fear of adjacent segment fracture as magnetic resonance (MR) images of T9 vertebral body were showing very thin posterior cortex and involvement of pedicles. Vertebral reconstruction was carried out by employing a thorough curettage via bilateral transpedicular posterior approach using autogenous iliac crest strut bone graft that is one of the cost effective alternative for cage in which both the endplates and disc need to be removed. Since the vertebral haemangioma was a benign tumour, the patient was not given postoperative radiotherapy.

Our technique has several advantages-Reduction of vascularity of tumor by alcohol injection (safe and easy to perform). Surgery by posterior only approach addressing decompression, curettage of entire tumor mass via bilateral trans-pedicular approach, adding tricortical bone graft to prevent further collapse of vertebral body and ad- 
dressing the adjacent segment collapse of haemangioma with thin posterior cortex in obese female patient with gross osteoporosis.

Postoperatively, motor power improved rapidly to $4 / 5$ in both lower limbs within 3 weeks and patient was mobilized with thoracolumbosacral orthosis. one-year follow-up, the patient is pain free and walking without support. CT showed no collapse of involved vertebra with complete resolution of edema on MR images.

In conclusion, this case report reveals that patients with multiple vertebral haemangiomas in noncontiguous vertebrae, who have had cord compression at one level with neurodeficit, can be operated on in two stages with preoperative absolute alcohol (ethanol) injection that reduces intra operative bleeding risk. Severe osteoporosis and adjacent segment problems need to be taken into account. Surgical decompression and tri cortical iliac crest bone graft with pedicle screw fixation constitute $360^{\circ}$ stabilization eventually leading to complete neurological recovery.

\section{Conflict of Interest}

No potential conflict of interest relevant to this article was reported.

\section{References}

1. Acosta FL Jr, Dowd CF, Chin C, Tihan T, Ames CP, Weinstein PR. Current treatment strategies and outcomes in the management of symptomatic vertebral hemangiomas. Neurosurgery 2006;58:287-95.

2. Fox MW, Onofrio BM. The natural history and man- agement of symptomatic and asymptomatic vertebral hemangiomas. J Neurosurg 1993;78:36-45.

3. McAllister VL, Kendall BE, Bull JW. Symptomatic vertebral haemangiomas. Brain 1975;98:71-80.

4. Obana Y, Tanji K, Furuta I, et al. A case of malignant transformation in thoracic vertebral hemangioma following repetitive irradiation and extraction. Pathol Int 1996;46:71-8.

5. Yang ZY, Zhang LJ, Chen ZX, Hu HY. Hemangioma of the vertebral column. A report on twenty-three patients with special reference to functional recovery after radiation therapy. Acta Radiol Oncol 1985; 24:129-32.

6. Heiss JD, Doppman JL, Oldfield EH. Treatment of vertebral hemangioma by intralesional injection of absolute ethanol. N Engl J Med 1996;334:1340.

7. Bas T, Aparisi F, Bas JL. Efficacy and safety of ethanol injections in 18 cases of vertebral hemangioma: a mean follow-up of 2 years. Spine (Phila Pa 1976) 2001;26:1577-82.

8. Lee BJ, Lee SR, Yoo TY. Paraplegia as a complication of percutaneous vertebroplasty with polymethylmethacrylate: a case report. Spine (Phila Pa 1976) 2002;27:E419-22.

9. Wenger M, Markwalder TM. Cement leakage and the need for prophylactic fenestration of the spinal canal during vertebroplasty. J Bone Joint Surg Am 2002;84: 689.

10. Doppman JL, Oldfield EH, Heiss JD. Symptomatic vertebral hemangiomas: treatment by means of direct intralesional injection of ethanol. Radiology 2000; 214:341-8. 